\title{
Grain deformability influence in the fracture behaviour of rock
}

\author{
N. Monteiro Azevedo* M. L. Braga Farinha ${ }^{+}$ \\ ${ }^{*}$ Concrete Dams Department \\ Laboratório Nacional de Engenharia Civil (LNEC) \\ Av. do Brasil 101, 1700-066 Lisboa, Portugal \\ e-mail: nazevedo@lnec.pt, web page: http://www.lnec.pt/organizacao/dbb \\ ${ }^{+}$Concrete Dams Department \\ Laboratório Nacional de Engenharia Civil (LNEC) \\ Av. do Brasil 101, 1700-066 Lisboa, Portugal \\ e-mail: lbraga@lnec.pt, web page: http://www.lnec.pt/organizacao/dbb
}

\begin{abstract}
Rigid particle models taking directly into consideration the physical mechanisms and the influence of the material grain structure have been developed for fracture studies of quasi-brittle material such as rock. Initially, the proposed circular/spherical rigid particle models were not able to match the ratio of the compressive strength to tensile strength that occurs in rock and the predicted macroscopic friction angle was much lower than the known hard rock experimental values. For this reason, several enhancements have been proposed to address these issues, namely the use of a clumped particle logic, the increase of the number of contacts per particle, the adoption of a polygonal/polyhedral grain structures.
\end{abstract}

With the same purpose, a 2D rigid particle generalized contact model (VGCM2D-Rigid) has been proposed [1] that properly reproduces the rock friction angle, the rock tensile strength to compressive strength ratio and the rock direct tensile to indirect tensile ratio, while keeping the simplicity and the reduced computational costs characteristic of circular particle models. More recently, the model has been extended to 3D [2]. Both particle models consider in an approximate way the polygonal/polyhedral grain geometry. The $2 \mathrm{D}$ and $3 \mathrm{D}$ particle models have been further extended to include in an approximate way the particle deformability by considering in each grain an inner finite element mesh (triangles or tetrahedra).

The 2D flexible particle model is tested against known experimental data on granite rock, namely biaxial tests and Brazilian tests. The obtained results are compared with those obtained with a rigid particle model with similar contact strength properties and with a flexible particle model, where the flexibility is due to the inner discretization of the grain with smaller particles. The analysis that is carried out shows that by including the particle deformability a more realistic hard rock macroscopic behaviour is predicted even if, for computational reasons, a simplified circular particle interaction is adopted. The results also show that the proposed 2D flexible particle model can predict a behaviour similar to a flexible particle model through inner particle discretization that is more computational demanding. It is also shown that when compared with a rigid model, the flexible model requires a smaller value of contact fracture energy to give a good agreement with known the experimental data.

\section{REFERENCES}

[1] N. Monteiro Azevedo, M. Candeias, and F. Gouveia, "A Rigid Particle Model for Rock Fracture Following the Voronoi Tessellation of the Grain Structure: Formulation and Validation", Rock mechanis \& Rock Engineering, 48 (2), 535-557 (2015).

[2] M. Candeias, N. Monteiro Azevedo and L. Farinha, "A 3D particle model for rock fracture based on the voronoi diagramas of the granular structure", Geotecnia, 143, 171-197 (2018). 